\title{
AN ONLINE-BASED VISUAL FRAMEWORK OF 3D POSITIONING DATA WITH WIRELESS SIGNAL FOR BURIED PERSONS
}

\author{
HYOUNSEOK MOON \& WOOSIK LEE \\ Korea Institute of Civil Engineering and Building Technology, Republic of Korea
}

\begin{abstract}
When a structure collapses during a disaster, many people are buried inside the collapsed building or debris. In order to detect the burrower, detection activities are performed directly in the disaster area using ultrasonic, acoustic and other detection equipment. Therefore, relieving the burrower in the early days of the disaster is the most important activity. The existing method requires time and manpower for the relief workforce to be put into the field after the disaster, and there is a possibility that many people may be injured due to inaccessibility and incorrect location data for buried persons within the Golden Time according to the situation in the field. The purpose of this study is to visualize the location information of the burrowers so that relief workers can quickly identify the location of the burial site, and to build a system development and operation framework that can be utilized in the online environment. The drones preferentially collect wireless signals (Wi-Fi) and air pressure information from barometer sensors, transmitted from mobile devices owned by the passengers through a route over the disaster area. The collected information is transmitted to the server on the ground using the disaster long term evolution (LTE) communication network. Then, the GPS position and depth value at the point where the wireless signal from underground is the strongest is recorded as the $2 \mathrm{D}$ position with $\mathrm{X}, \mathrm{Y}$ coordinate and $3 \mathrm{D}$ position with $\mathrm{Z}$ coordinate of burial depth for buried person. The final position value for each person can be displayed visually with signal strength values in the open map. This information is expected to be used as an important tool for relief workers to quickly identify the precise location of the buried person and to prepare the relief plan in advance.

Keywords: wireless signal, buried person, 3D positioning, visual framework, detection.
\end{abstract}

\section{INTRODUCTION}

The securement of early disaster response technology in the event of the large-scale collapse of urban buildings such as Sampung Department Store in Korea (1995), and a national disaster such as Nepal earthquake (2015) and Chilean mine collapse (2010) plays an important role in securing the life golden time for buried persons. In this way, the provision of location information through rapid and accurate location detection of the buried person at the collapse site can be utilized as an indispensable technology for rescue in the event of a disaster [1].

In order to detect the buried persons of the formerly collapsed area, it is mainly used to search for and relieve the buried persons by bringing in rescue dogs or conventional detection devices [2]. However, since this detection method is directly executed to the upper part of the collapse site by a relief workforce, there is a risk of additional loss of life due to the second collapse. In addition, there are disadvantages in that it is difficult to operate due to complex use of detection equipment, and it takes a long time to detect it. In addition, without the direct input of the equipment in the field, it is difficult for the buried person to roughly identify the buried position and their status in real time from outside the collapsed place.

In the previous researches, the Wi-Fi Radio Map [3], [4], of the indoor space was constructed rather than the location visualization of the buried person, and the heat map [5], was constructed according to the distribution of the signal intensity. A similar study has been conducted in the Lausanne University of Technology (EPFL) [6], in Switzerland where 
research on missing person detection through the drones has been attempted. However, there is a case where only a simple detection location is displayed rather than a visualization of the multiple detection location according to signal strength. It is difficult to check the condition of the portable device and the signal characteristics of the burial position of the buried person only by using such location information. It also has limitations in determining the relief priorities of the buried person. The visualization information of the detection location provides the analysed result according to the characteristics of the signal intensity and can be utilized as the meaningful location information of the buried person.

To solve these problems, we constructed a methodology to detect the location of the buried person in the remote site with the detection issues of the buried person using the drone (UAV; Unmanned Aerial Vehicle). In this study, the conceptual positioning methodology of the location of the buried person using the drones is discussed, and the integrated framework of how to provide the burial location information through the visualization of the burial location information is developed based on this concept. Therefore, a visualization framework based on the location information of the buried person was constructed, and a system mechanism for data transmission and processing between the burial detection module installed in the drone and middleware in the ground was constructed. First, we set the communication protocol of wireless data transmission for system design. Then, an agent server (Middleware) that primarily stores and processes the received data is designed, and the $\mathrm{DB}$ (Database) is designed so that the processed data can be classified and managed. Using the data table information stored in the DB, the signal strength and location information were extracted, and the color scheme for each signal strength was constructed, and the Google and Naver Map API was used so that only the location information having the highest signal could be displayed on the online map.

In future, this system will be widely used as a core relief tool to provide information on the location of missing persons as well as to locate the buried persons.

\section{3D POSITIONING FRAMEWORK FOR BURIED PERSONS}

Fig. 1 shows an integrated framework that implements rapid detection and visualization information system using drone in the collapsed area. In the event of a disaster, a drone is equipped with a wireless sensor-based lifeguard detection module are placed on the collapsed area to scan the mobile phone's Wi-Fi signal in the collapsed area. Then, it receives location information, signal strength, time, and pressure data from each mobile phone and transmits it to a human detection system in a real time through LTE communication network. After that, through the agent program and the DB server, the location of each mobile phone is visually represented on the online web map together with the signal strength through the open API interconnection of the Google and Naver from the web server. When the information is linked to mobile, the location information is realized in real time on the field, and it can serve as a navigation from the position of the relief manpower to the position of the burial person. This system is a tool for accurate rescue and lifesaving within life golden time through rapid location detection of the buried person. In the next section, we describe the schematic mechanism of three-dimensional location positioning, including two-dimensional location and depth values, processed from the burrower location detection module mounted on the drones.

Even if the buried persons in the collapsed terrain owns a portable device, the strength of the radio signal transmitted from the portable device in the collapsed debris is inevitably weakened. And some of them are transmitted through the cracks of the collapsed debris and some are emitted to the ground through diffraction or reflection. Also, since the signal 
intensity is plotted in a log scale against the distance, the test result shows that the signal can be detected up to $60 \mathrm{~m}$ in the open space. In particular, Wi-Fi signal strength can be confirmed only on the flat surface, and the GPS coordinate value when measuring the strongest signal strength is recorded to estimate the two-dimensional plane position of the buried person.

Since the estimation of the burial depth can theoretically be measured by wireless signals alone, it cannot be confirmed at actual measurement, so the $\mathrm{Z}$ value of the buried depth can be estimated through fusion with the barometer sensor mounted on the portable device. The pressure information can be estimated more accurately by simultaneously using the air pressure value measured by the portable device and the external pressure information according to the climate of the measurement day and the time zone of the area. Especially, the probability of detecting the wireless signal of the deepest buried person is low when the position of the buried person with different burial depth is measured by the signal strength. In this case, if the signal of the deepest burrower is transmitted directly to the portable device of the buried burrower at the upper position, and it is possible to transmit the signal strength to the burrower, simultaneous estimation of many burial locations is possible regardless of the depth. The measured signal intensity, Mac address, GPS position, and depth value by atmosphere pressure are transmitted to the ground site through wireless communication, and the corresponding position value with signal strength is visually displayed on the $3 \mathrm{D}$ collapse terrain information constructed by the drones. The final goal will include a decision-making function that can determine the area to be relieved first by identifying a large number of buried persons and burial areas.

\section{A VISUAL FRAMEWORK OF LOCATION INFORMATION FOR BURIED PERSONS}

In this study, drones were used for rapid detection for securing the life golden time of the buried persons in the collapsed area. Since it is difficult to detect the burrower with the drone alone, the detector module for buried persons capable of carrying out the flight control of the drone was developed and installed to detect the portable device possessed by the burrower.

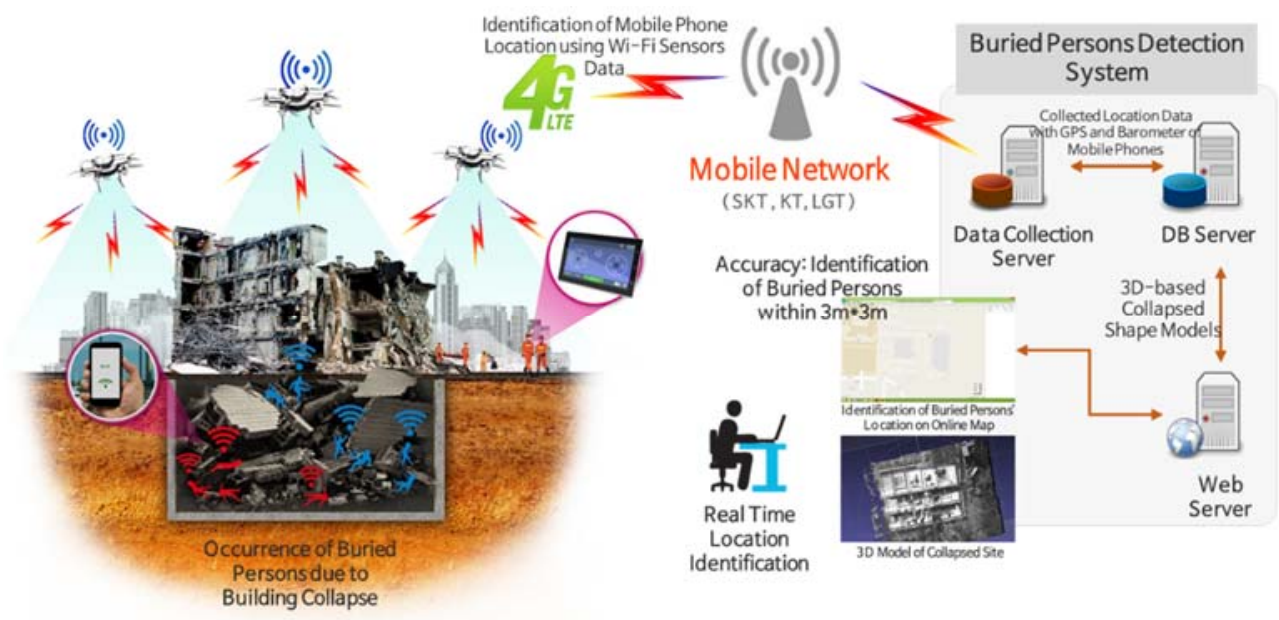

Figure 1: An integrated framework for detecting buried person using UAV. 
This module continues to detect the signals of portable devices during the flight to the top of the collapsed ground, collects various sensor information in real time, records the position of the strongest signal point and transmits it to the ground server. This system is used to acquire, store, analyse, transmit, process, and visualize data, and in the next section, an online-based location visualization framework is constructed.

\subsection{An online-based location visualization framework for buried persons}

The embedded location visualization system developed in this study is implemented through the following online based visualization framework. It is possible to acquire data that can detect the position of the buried person more precisely by improving the H/W performance of the existing first developed detection module and adding the pressure information value. By using this, the position and signal strength of the buried mobile phone are displayed on the two-dimensional Google map and the Naver map, thereby realizing the transmission and processing system for displaying the position data in the two-dimensional image of the burial area.

In the system conceptual diagram below, the processing path of the whole data can be known, and the communication protocol is specified between the prototype of the detection device of the drones and the agent server, and each data is collected. Then, the data is gathered and stored in the DB server. When executing the web viewer program, the value stored in the DB is displayed on the screen together with the visual information of signal intensity through the open API of Google Map and Naver Map.

\subsection{Development environment}

This system is connected to the mobile device whenever the Internet is available, so that the position of the burial person can be visually realized according to the signal strength through the web page or the position of the buried person having the highest signal can be intuitively identified. JAVA JDK 1.7 is applied to web development language to enable operation in online environment. The database is rather heavy, but ORACLE $11 \mathrm{~g}$ has been configured to ensure the stability of real-time processing of massive immobilizer signals and GPS data. The connection of the database used OJDBC 6.0.JAR. Google Chrome was chosen as the optimized implementation browser to improve its stability and compatibility with online maps. It can be accessed in other browsers. The server IP is initially set to 118.47 .55 .56 and the communication server port is assigned to 9090 .

\section{A SYSTEM ARCHITECTURE DESIGN}

In the system design stage, we specified communication protocol, agent (middleware) design and database design. After designing the system core, we adopted UI (User Interface) of web design for displaying processed result in online environment, and configured intuitive and easy UI based on user's usability to be connected in mobile environment.

\subsection{Definition of communication protocol}

In this section, we have defined the protocol for communicating between the human detector module mounted on the drones and the agent server on the ground. The communication server uses port 9090 and the basic server connection DNS is set to 118.47.55.56. Web access is possible only with this DNS. 


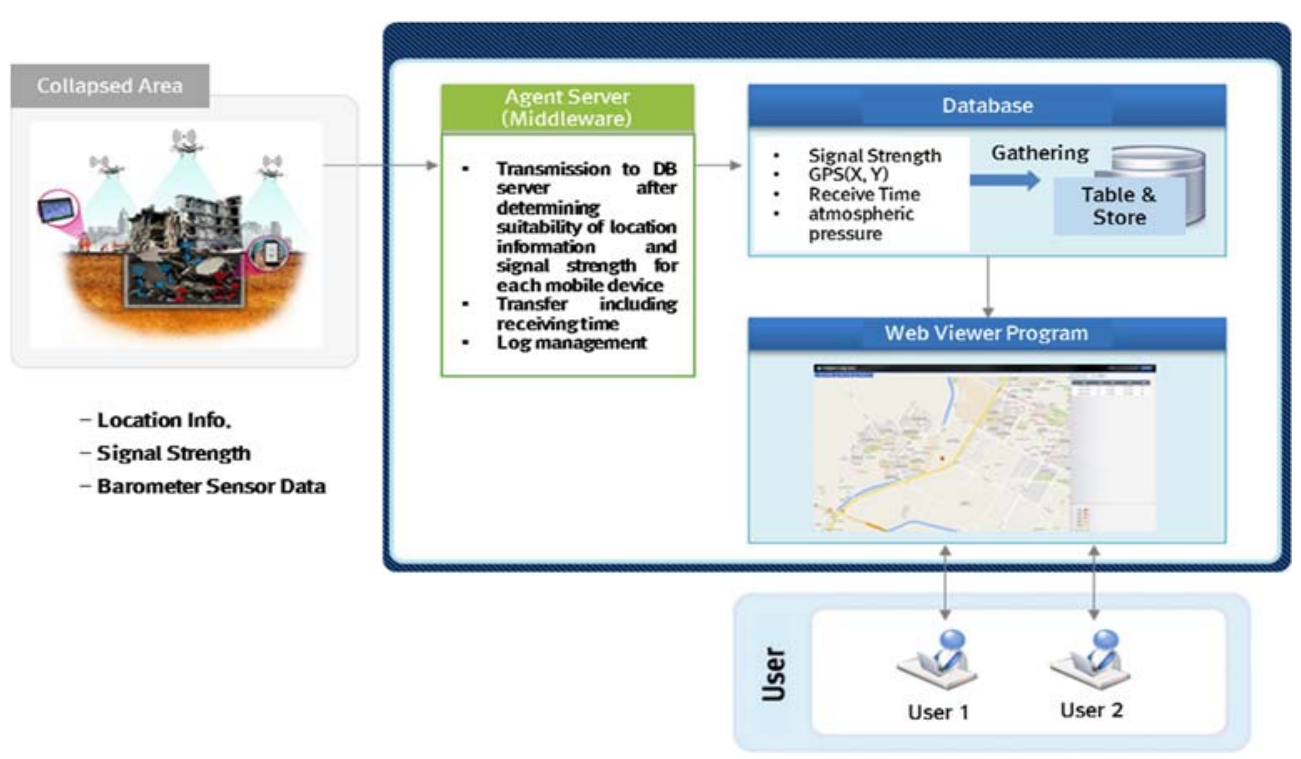

Figure 2: An overall system mechanism for configuring location for buried persons by UAV.

\section{Communication Packet (WCDMA Transfer)}

- Basic Packet Structure
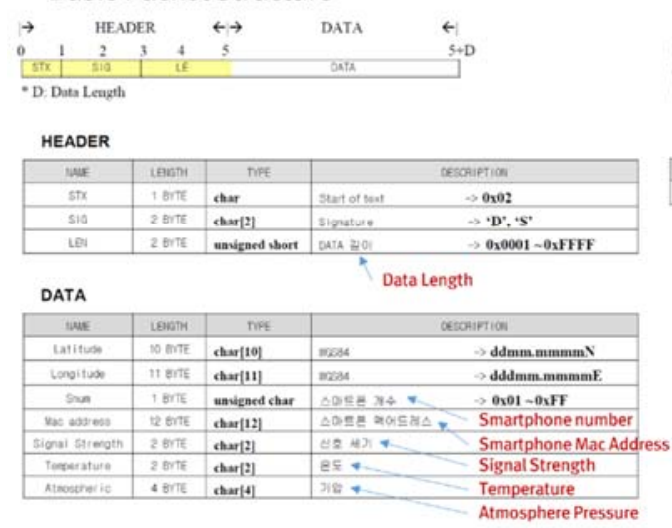

\section{Communication Packet (WCDMA Receive) \\ - Basic Packet Structure

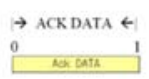

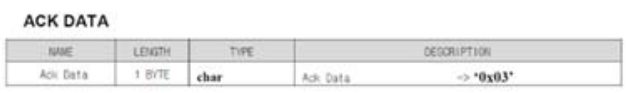

Figure 3: Definition of communication protocol for transferring and receiving data.

In the definition of the communication protocol, the structure of the data transmission packet transmitted by the portable device is defined, and the data receiving packet structure received by the portable detection module is defined. The transmission packet is divided into Header and Data, and the header defines the basic type of data packet to be transmitted. Data is composed of data packet that is the key to location of the buried person with which includes data structure to be transmitted by mobile device. This case includes the latitude, longitude (WSG84) indicating the two-dimensional coordinate system, Snum indicating the number of the smartphone, Mac Address indicating the received signal strength of the smartphone 
wireless signal, the signal strength of each smartphone, Atmospheric indicating temperature and sea level pressure value for the barometric correction according to the weather condition.

If Snum is "0x02", it means that Mac address and Signal Strength are transmitted twice. That is, since two smartphones have been detected, you can tell them to transmit two Mac addresses and signal strength. The data receiving packet has a basic packet structure of ACK DATA. In accordance with this communication protocol, it is responsible for communication packet processing to the buried mobile device, the human detector module of the drones, and the ground agent to realize the burial location visualization data.

\subsection{Agent server (middleware) design}

The agent is a kind of middleware type tool that processes the data acquired from the human detection module to receive it from the server and utilize it as the detection location information for buried persons with reliability and integrity.

The detection module and its detection information are transmitted to the ground via the TCP (Transmission Control Protocol) service, and these pieces of information are input into DB information. Also, the human detection module transmits information such as latitude, longitude, the number of mobile phone, Mac address, and signal strength to the server according to the TCP communication protocol. The server accesses the human detection module through the Internet environment and collects the module header information to check the accuracy of the module information. Then, the validity of the length value of the module information is checked and conversion to HEX data is performed through data analysis. In the data analysis process, ACK DATA, which is a received packet, is processed, and this data is transmitted to the human detection module so that the detected information can be stored according to the number of receiving apparatuses. Through this iterative process, detection information such as final burial location information and signal strength is collected and registered in the predefined Oracle DB. Fig. 5 shows the intensity of Wi-Fi signals detected by the human detection module and the GPS coordinates packet of the module in real time, and the processing result of completing the data transmission to the DB is implemented.

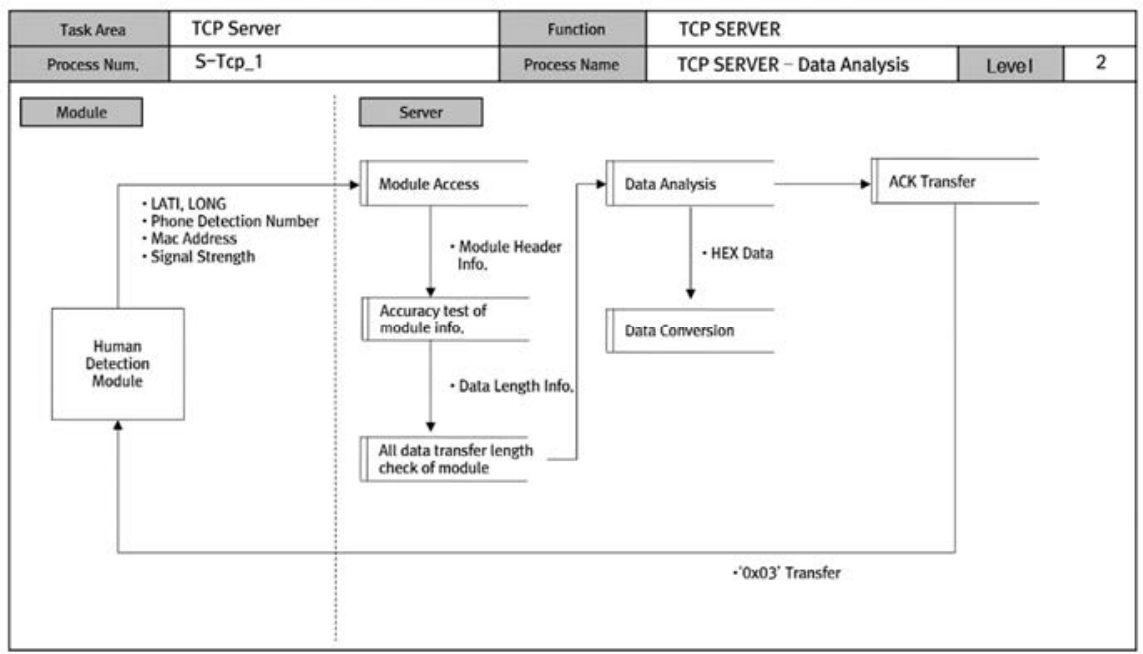

Figure 4: Process design of agent server (middleware). 


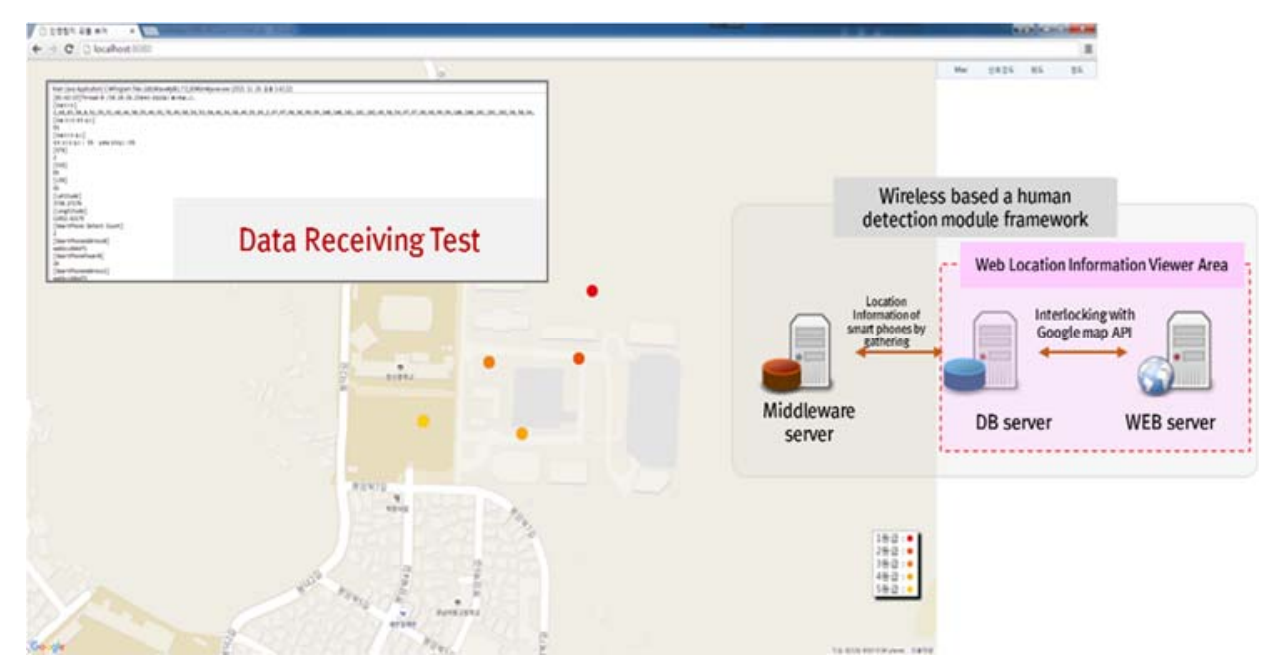

Figure 5: Data transmission validation test by agent server with the collected data.

\subsection{Database design}

We constructed the database model to construct the final data storage system processed through agent. The DBMS used is Oracle $11 \mathrm{~g}$ and the user space is allocated as $30 \mathrm{MB}$. The user space of the DB is composed of the user LIFESAVER that accesses SYS (Operator) which is the database administrator account information and the DB of the detection module. The physical database model generates the LIFE table and generates seven records, MAC representing the portable device of the buried person, SIGNAL representing the signal strength, LATI being the $\mathrm{X}$ coordinate value, LONGI being the $\mathrm{Y}$ coordinate value, BARO being the air pressure value from the collected barometer sensor, DEPTH, which is the depth value calculated from the value, and REGDATE, which indicates the recording time. Here, LATI and LONGI are recorded by GPS coordinate values.

In this DB configuration, the Wi-Fi signal strength received from the agent program and the GPS coordinates can be displayed in the following figure as a tabulated table that can be easily displayed in a web program. The GPS information can be recorded in 5 seconds as an initial value, but can be set in units of 1 second by user definition. Due to the nature of GPS, location information can be gathered up to 1 second.

\subsection{Location visualization colour scheme by signal strength}

In order to determine the position of the buried person, it is necessary to use Wi-Fi signal strength and GPS data of the collected portable device as core information. It is difficult to obtain accurate position information according to the signal strength of the detected portable device. The drones' human detection module records GPS information along with Wi-Fi signal information. From these information, the GPS position value at the point where the signal is strongest among multiple position values from Mac Address of the portable device is recorded. When the position value is displayed on the open map, the signal having the strongest position value is filtered and displayed on the screen. As a mark-up colour 


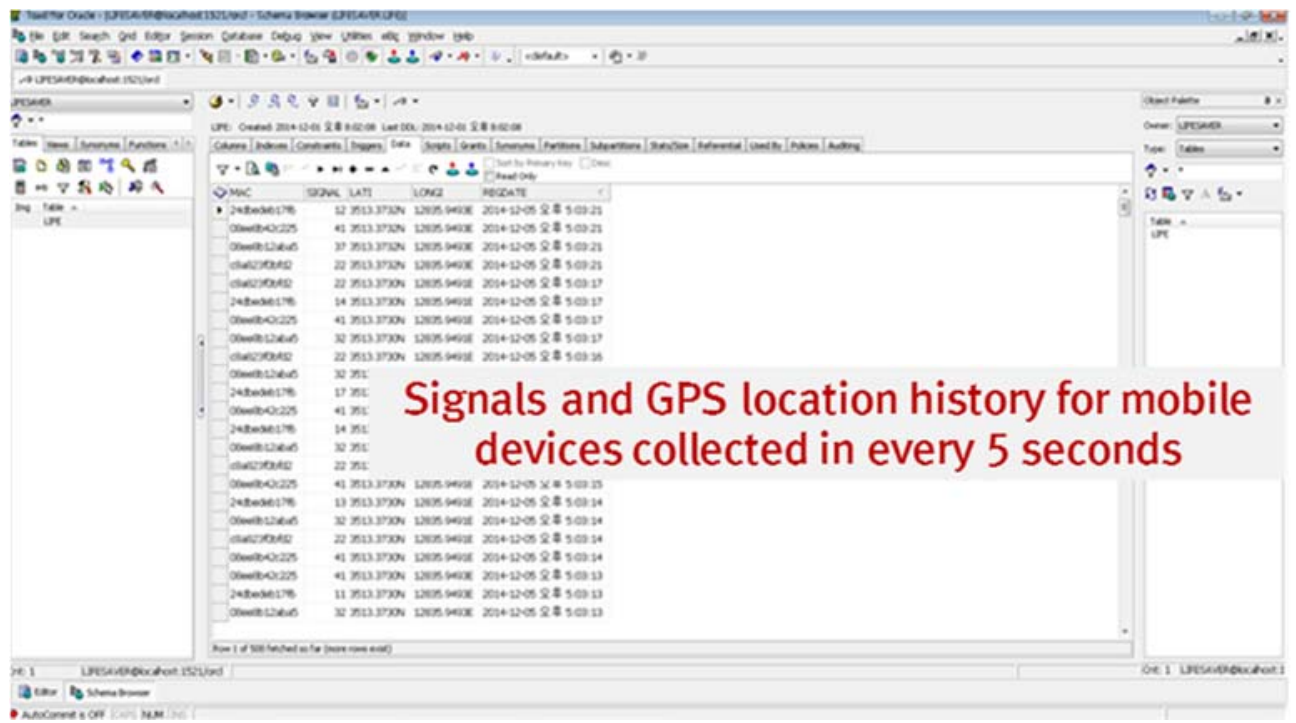

Figure 6: Result test of initial data processed by agent server with DB.

\section{$5 \quad 10152025303540455055606570758085$ 100000000000}

Figure 7: Colour scheme by signal strength.

scheme of the location, the colour spectrum for each signal intensity is constructed as shown below. Actual signal strength is represented by - value, but it is converted to + value in order to improve understanding of signal strength. In other words, the higher the number, the higher the signal strength.

\section{DEVELOPMENT AND TEST OF WEB-BASED VISULATION SYSTEM}

In this section, we have developed a location visualization program with supporting Google and Naver map that can access the web through the development of the online-based location visualization framework for buried persons described above. In order to test this, a collapsing training site similar to the actual one was selected, and the process from acquisition of location information to visualization presentation was tested for five mobile devices.

\subsection{Development of online-based location visualization system for buried persons}

The system is developed to visualize the location information of the burial mobile devices collected from the human detector module installed in the drones and to provide quick decision-making information to relief workers. It is possible for this system to connect from the Internet access. This system has been optimized for the Chrome browser. In order to have versatility, this system applied Google map and Naver map which is general purpose web map in Korea. The system includes a switch between Google map and Naver map, a visual 
representation by signal strength of mobile device, receiving history of Wi-Fi location recorded at one point, information transmission period setting, and location visualization display by map. In addition, the log file of recorded location information for analysis can be downloaded to an Excel file (*.xls), and the function of deleting the previous record for detecting the location of the new disaster area was also included. Here, the update period of the position of the buried person can be displayed in units of 1 second according to the GPS recording unit, but it is possible to customize the update period in real time up to 0.1 second.

Fig. 8 shows the main screen of the developed location visualization system for detecting the buried person. The current map screen shows the Naver map screen. In the center of the screen, the position value of four mobile device carried by four persons is visualized by filtering only the strongest radio signal. You can select the location on the screen and see what information is collected on the right side of the screen. You can check the Mac Address, signal strength, recorded GPS value and air pressure information for the mobile device at the selected location. First of all, it is possible to confirm the plane position by GPS value only and to obtain approximate depth value by altitude conversion of air pressure value.

Also, if you select the given location and select the Wi-Fi History button on the main screen, you can check the record of the position value by each signal of the buried mobile device recorded according to the planned path of the drones on that location.

From these results, only the position having the maximum signal value is filtered and displayed. For your reference, the above system can be confirmed through http://118.47.55.56:8080/life/main address access, but it is restricted to access through administrator login. In the future, we will expand the functions to be utilized in various fields through registration of users.

\subsection{Field test and verification}

The team selected a site similar to the actual collapse area to test the developed online-based location visualization program for buried person. This site is a training ground temporarily built for firefighters to relieve the buried person in the collapsed area.

First, mobile devices of different manufacturers are tied to strings and installed at the site from the top of the collapsed terrain to the buried ground with varying burial depth. Then, a human detection module was installed in the drone so that it can be detected at a predetermined time interval according to the planned path inside the collapsed area. Setting

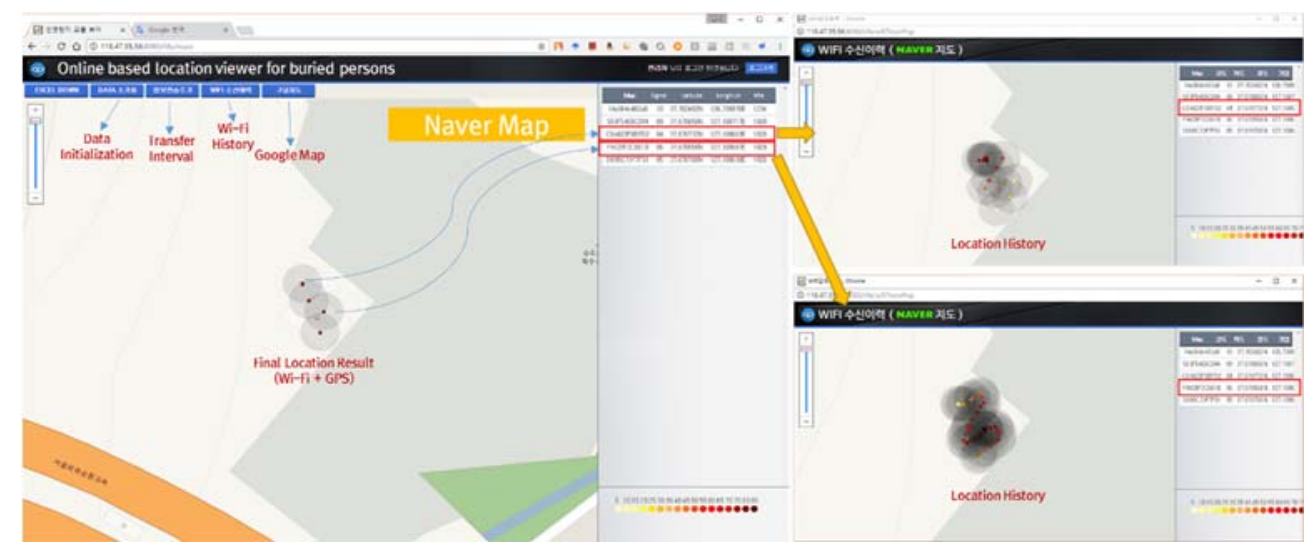

Figure 8: A main screen with location visualization result for a collected data. 
the collapsed zone with the boundary line for detection will automatically create a route plan, and the Autopilot feature in the drone will fly according to the specified path and speed. At this time, the speed can be pre-set by the user, but it is set at a speed of $5 \mathrm{~m} / \mathrm{s}$. However, in a position where high signal intensity is generated in the movement process, the drone hovering is activated to collect a large number of signal information from the underground, and the position information is recorded together with the signal strength information every 1 second as a GPS information recording unit. Afterwards, the signal of the portable device in the collapsed site is automatically stored in the memory of the human detection module mounted on the drone in real time. The LTE network or Wi-Fi is used to transmit the stored data to the ground site, and visualization is performed through the processing of the collected data configured through the above-mentioned ground server.

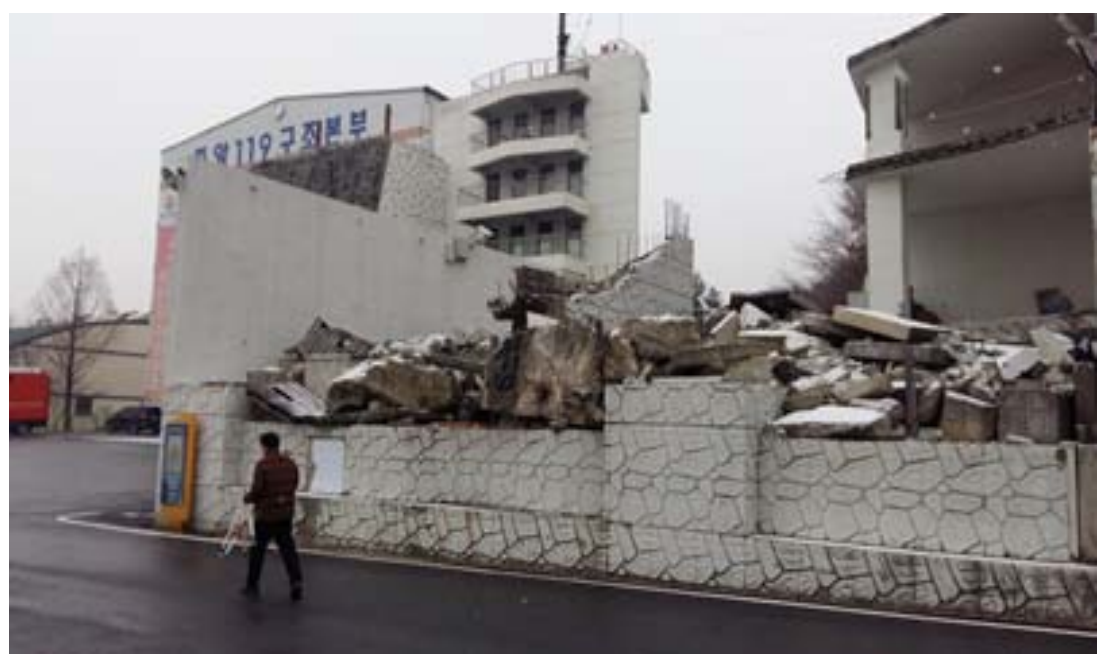

Figure 9: Field test site with collapsed site for firefighters in Korea.

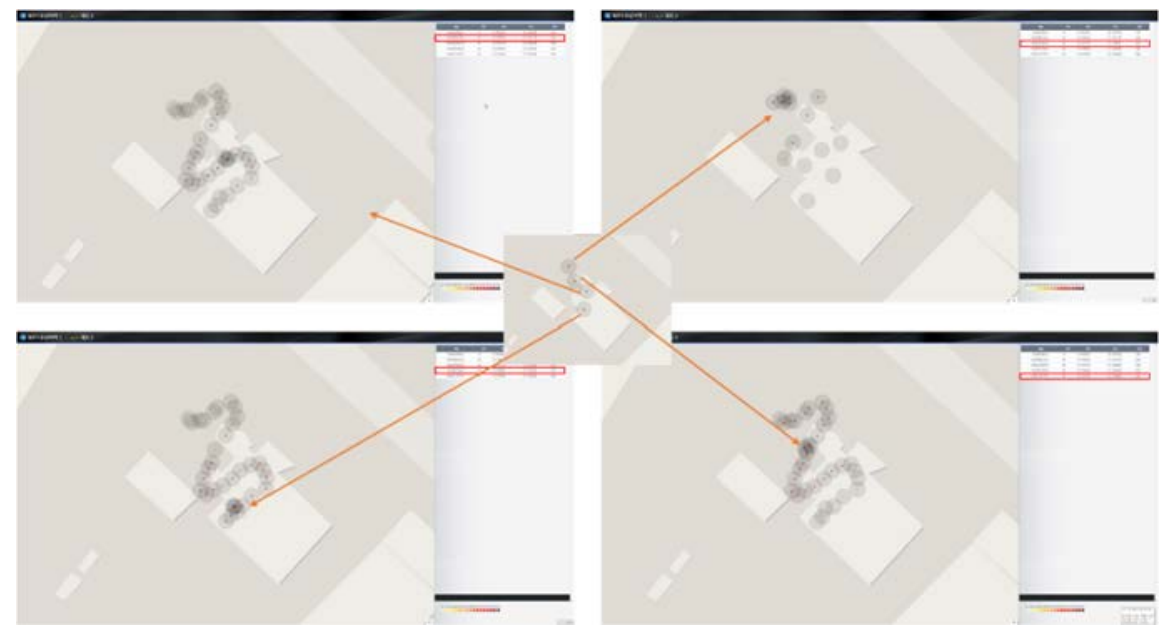

Figure 10: Test result for 4 mobile phones of the virtual buried persons using drone. 
Fig. 10 shows the location visualization result for mobile phones in the online map by processing the final acquired information from the agent server by performing actual field test. According to some map types, the coordinate system does not fit. For example, Google map does not provide accurate map in Korea, so the error between actual position and measured position is more than about $5 \mathrm{~m}$. However, since Naver map provides domestic precision map, the error with actual map is less than about $1 \mathrm{~m}$. As shown in the figure, the actual position of one buried person is extracted from the most powerful position from the plurality of positions and signal strength information obtained according to the path. The location of the actual buried mobile device was precisely derived from each location through GPS measurement. The mean error value was measured through the mutual comparison with the coordinate values displayed on the web map after the drone detection.

As a result, it has an error range of about $5.8 \mathrm{~m}$. If it is an actual collapse site, it is a somewhat large error for detection range and it is difficult to recognize it as a precise location. In this case, an algorithm for analysing the optimal position by acquiring a lot of position information for a single mobile device buried needs to be developed, and a probability density function corresponding to the distance from the actual position should be calculated.

\section{CONCLUSIONS AND DISCUSSIONS}

In this study, the location of buried persons in the collapsed area is detected, and the acquired information is processed to visualize the location on the online map together with the signal information to provide a tool for decision making of the relief manpower. For this purpose, this study constructed the information transfer mechanism and data processing system collected from the human detection module installed in the drone, and developed an online map-based burial location visualization system for the buried persons to visualize such data.

The system developed in this study can be used not only to locate buried persons but also to provide location information more accurate than existing Cell-ID methods by tracking the location of mobile devices of missing persons. In addition, the tested site is similar to the actual site, but it contains somewhat open space, so no tests were conducted under extreme burial conditions. In addition, it is possible to acquire the actual atmospheric pressure value at the corresponding position from the barometer sensor of the mobile device, but it is necessary to convert it to the altitude value considering the weather of the relevant area. Especially, it is required to improve the system that expresses the result of calculating the optimal position detection probability by analysing a plurality of signal values per position measured from a single portable device. Especially, the human detector module installed in the currently developed drones has low Wi-Fi detection performance and it is difficult to search multiple devices simultaneously because the number of channels of the wireless signal that can detect at a time are small. Therefore, the firmware of the Wi-Fi module core needs to be replaced with the high performance based on real time OS (Operating System). In addition, it should include the coordinate transformation algorithm based on the map transformation, and it will be necessary to link with the acquisition of the $3 \mathrm{D}$ collapse terrain to confirm the depth of burial site.

This technology includes not merely a web program that can be used in a disaster situation, but a system developed through fusion of drones, human detection modules, communication and transmission technologies. In future, this technology will be used as a decision-making system for estimating the clear location of the buried person in various disaster situations and it will be an important tool to secure the life golden time of the buried person. 


\section{ACKNOWLEDGEMENTS}

This study was supported by "(17Main-Big2-Convergence) Development of Human Detection and Emergency Relief Technology in Collapsed Site of Underground in Urban Area" project funded by Korea Institute of Civil Engineering and Building Technology (KICT).

\section{REFERENCES}

[1] Moon, H. \& Lee, W., Development and Verification of a Module for Positioning Buried Persons in Collapsed Area. Journal of Korea Academia-Industrial Cooperation Society, 17(12), pp. 427-436, 2016.

[2] Moon, H., Kim, C. \& Lee, W.,Wireless Communication-based Buried Person Detection Considering Characteristics of Collapsed Surface in Disaster Area. Proceedings of 2015 Civil Expo and Conference, 2015.

[3] Jeong, I. et al., A study of Establishment on Radiomap that Utilizes the Mobile Device Indoor Positioning DB Based on Wi-Fi. The Korea Society for GeospatIal Information System, 22(3), pp. 57-69, 2014.

[4] Kaemarungsi, K. \& Krishnamurthy, P., Modeling of Indoor Positioning Systems Based on Location Fingerprinting. Proceedings of Twenty-third Annual Joint Conference of the IEEE Computer and Communications Societies, pp. 1012-1022, 2004.

[5] Sagari, S. et al., Coordinated Dynamic Spectrum Management of LET-U and Wi-Fi Networks, 2015 IEEE International Symposium on Dynamic spectrum Access Networks, pp. 209-220, 2015.

[6] Carron, C., A Drone That Finds Survivors Through Their Phones, https://actu.epfl.ch/news/a-drone-that-finds-survivors-through-their-phones/. Accessed on: 5 Feb. 2017. 